\title{
$\mathfrak{S} \mathfrak{t} \mathfrak{a} \mathfrak{t} \mathfrak{a} \mathfrak{t}$
}

Des

\section{bentiden Suritentages.}

\section{$\S 1$.}

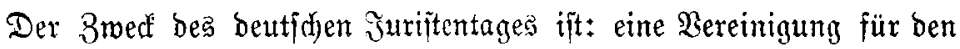
lebendigen Meinungsaustaufh uto ben perfönlidgen Berfehr unter ben beutichen Suriften zu bilden; nuf den Gebieten bes Privatredtas, bes Procefifess und bes Strafred)t

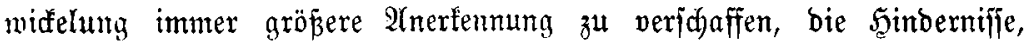
welche biejer Entwiffelung entgegenjtehen, zul bezeidynen und fid über $\mathfrak{B}_{\mathfrak{o r}}=$

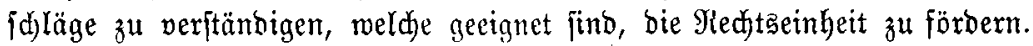

\section{$\S 2$.}

Der beutfdje Suriftentag tritt in ber Regel alliährlid zufammen, bod) ift bie jtänbige Deputation ermädtigt, aus Brünben ber 3med̆

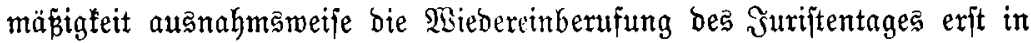
bem auf beffen leb̨ten Zllfammentritt folgenden zmeiten Ralenderjahre vorzunehmen.

\section{$\S 3$.}

3ur Mitgliebinaft beredjtigt find bie beutjuen Midter, Staatsan=

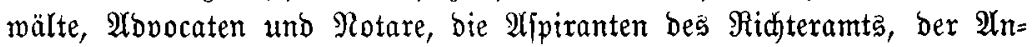
waltfdyaft und bes Rotariats, fowie jeber, Der nad) feiren \&andesgejetzen

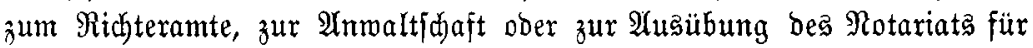
befähiggt erfannt ijt, ferner bie Rehrer an ben beutjden Şodjifulen, bie

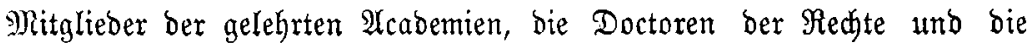
red)tagglebrten Mitglieber ber Berwaltungabehöroen. 
$\S 4$.

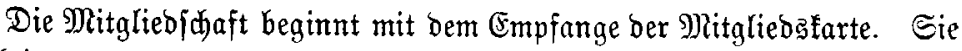
beredtigt zur Theilnahme an ben Berbandlungen und an der 26 fftimmung.

$\S 5$.

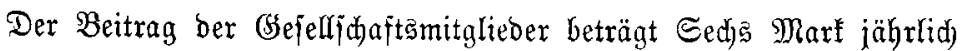

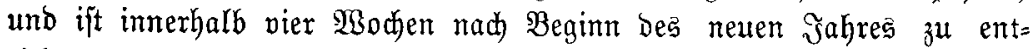
ridten, wibrigenfalfs berfelbe durd) Poftuoridü eingezogen mirb. Nimmt ein Mitglied ben mit Poitoorfdup befdmerten Brief nidft an, jo wiro

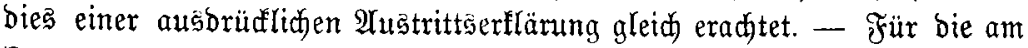

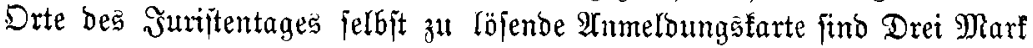
zu entridjten.

\section{$\S 6$.}

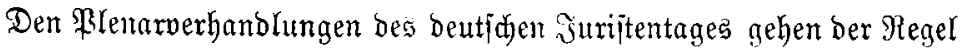

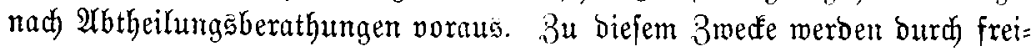
willige Einzeidumungen ber Mitglieber folgenbe vier Abtheilungen gebilbet:

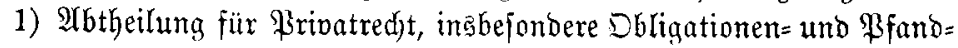

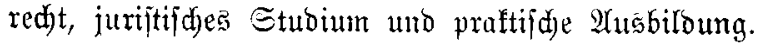

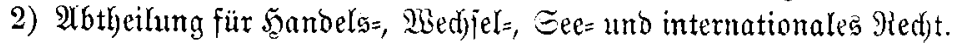

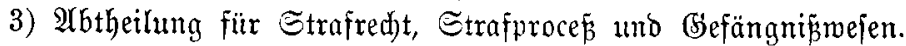

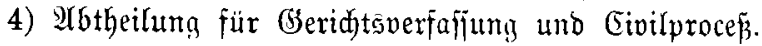

Die Âtheilungen wählen ihre Soriłbenden, Sd)riftführer, Șeridgt= exftatter umb benadfridfigen ben Boritzenden ber SYlenarverfammlung $(\S 7)$, fobals ihre Berathungen uber einzelne Gegenitände gefdlojien

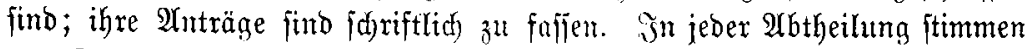
mur Diejenigen mit, weldhe fidy in bie betreffende 2(btheilung bereits ein = gezeidjuet haben.

Sämmtlidye Befdlitffe ber Mbtheilungen merben in der \$lenaroer= fammlung mitgetheilt. (Es finbet jebod) eine (Erörterting und Entfd)etoung im \$lemum nur bann ftatt, wenn biefelbe von ber betreffenden 2 (bthei= lung vorgefthlagen, ober wenn fie won mintoejtens zehn Mitgliebern be= antragt und von ber Fienarverjammlung bejolinfen mirb. Heber bie Borfrage, ob bem von mindejtens zehn Mitgliebern gejtellten antrage auf Plenarentfdideibung ftattzugeben, wiro nur einem ber $\mathfrak{A n t r a g j t e l l e r ~}$ und bent Beridjteritatter bas $\mathfrak{B}$ ort ertheilt.

\section{$\S 7$.}

Die Berhandlungen ber \$lenarverfammlung leitet ein Borfił̧ender, weldjer für bie Dauer eines jeden Juriftentages in ber erften \$lenarver= fammlung burd Stimmzettel oder Arclamation gewählt wirb. Derfelbe 
benennt zmei bis vier Stellvertreter und vier Sdriftführer. Er bejtimmt bie Tagesoronung und fann eimżelne Begenftände, ohne Borberathung in ben Arbtheilungen $(\S 6)$, unmittelbar zur \$lenarberathung ftellen. 2 Iud

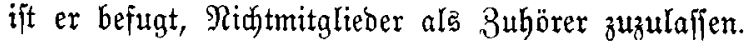

$\S 8$.

Bei allen Befdulüfien ber Blenarverjammlung und ber 2 (btheilungen entid)eibet einfadje Majorität ber anmefenden Mitglieber, bei allen $\mathfrak{B a h l e n}$ relative Majerität und im Falle bar Stimmengleidhheit bas 200 s.

\section{$\S 9$.}

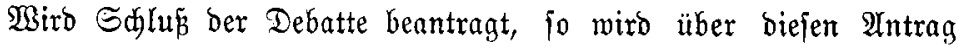

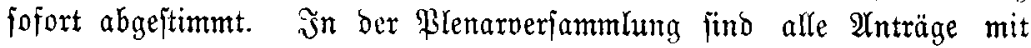

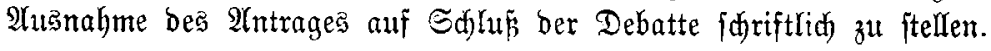

\section{$\S 10$.}

Bor bem Srhluije eines jeben Juriftentages miro von ber Plenar= verjammlung burd) Mcclamation ober in einem einzigen Scrutinium burd) Etimmzettel eire aus neunzzehn Mitgliedern und dem \$räjibenten bes leb̧ten Surijtentages als Šbrempräjibenten bejtehende ftändige Deputa=

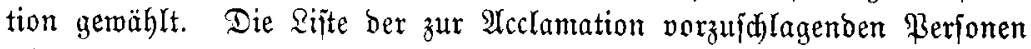

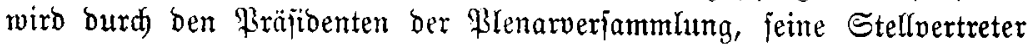
unb je zehn von jeber 2lbtheilung gewählte Bertrauensmänner gemein= fäaftlidid fejtgejtellt.

Die ftändige Deputation hat folgenbe Befugniffe und Dbliegenheiten:

1) fie jorgt für bie $\mathfrak{U}$ uafüh

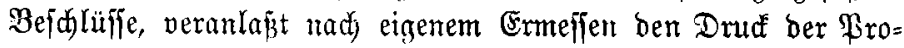
tocolle und Borlagen, bemirft bie Bertheilung ber Drudfacten an bie Mitglieder und vermahrt alle Ŷtcten und Sdiriftitüfe dę Эuriftentages ;

2) Fie bejtimmt 3eit und Drt bes nädjuten Şuriftentageŝ, trifft bie für benfelben nöthigen Sorbereitungen, erläp̆t bie Einlabungen, ftellt bie vorläuftige Iagesoronung auf, mobei fie in ber Regel mur bie bis jum 31. Mai bes laufenden Эahres eingegangenen

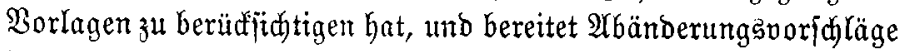
in Betreff der Gefdäfțoronung für bie \$lenarverjammlung vor;

3) fie nimmt bie Beitrittserflärungen neuer Mitglieber entgegen, fer= tigt bie Mitgliebśfarten aus, empfängt bie Beiträge, beftreitet

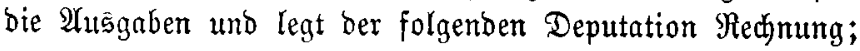


4) fie ergänzt fid felbit, falla eines ober mehrere Mitglieder währeno bes Giejđäftajahres aubjheiben.

Die Deputation wählt aus ihrer Mitte einen Boriłzenden, einen Sdrifftführer, weldher ein von der Deputation feitzufekendes \$aufdoquan=

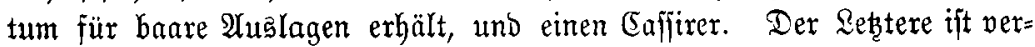
pflidłtet, Ser ftänbigen Deputation bei ifrem jebesmaligen Bulammentritt einen Eaffenabjdlup vorzulegen. Die Deputation läft burch eines ober

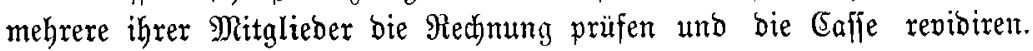

Die Deputation beftimmt Drt und Beit ihrer 3ufammenfunft. Zur

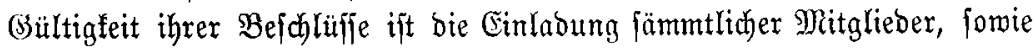
die Mitwirfung von wenigftens fünf Mitgliebern erforberlid.

\section{$\S 11$.}

Abänderungen biejes Etatutš fönnen z̧war von ber \$lenarverfamm= lung burd) einfache Stimmenmehrbeit, jeood) nur auf (d)riftlid)en 2 ntrag, Der vier 230 hen vor bem 3 úammentritt bes J̃urijtentages ber ftändigen Deputation (\$ 10) überreidyt worden, bejdloffen werben. 